\title{
Análise de lesão renal em pacientes de terapia intensiva com diagnóstico de COVID-
}

\section{9 em uso de antibióticos}

\author{
Analysis of kidney injury in intensive care patients diagnosed with COVID-19 using antibiotics \\ Análisis de la lesión renal en pacientes de cuidados intensivos diagnosticados de COVID-19 \\ mediante uso de antibióticos
}

Recebido: 20/12/2021 | Revisado: 27/12/2021 | Aceito: 04/01/2022 | Publicado: 06/01/2022

Tatiele Quiquinato Viana ORCID: https://orcid.org/0000-0001-5825-1396 Hospital Regional de Mato Grosso do Sul, Brasil E-mail: taty_quiquinato@hotmail.com.br

Paula Juliani Nascimento Rodrigues ORCID: https://orcid.org/0000-0001-6224-6311

Universidade Anhanguera-Uniderp, Brasil E-mail: paula.juliani@educadores.net.br

\begin{abstract}
Resumo
O objetivo dessa pesquisa foi caracterizar o perfil dos pacientes que desenvolveram LRA, analisar os exames laboratoriais, classificar de acordo com os estágios e analisar o ajuste de posologia dos antimicrobianos. Trata-se de um estudo transversal, analítico e retrospectivo, cuja coleta de dados foi realizada com informações contidas no Sistema de Prontuário Eletrônico MVPEP®. No período de estudo, foram encontrados 100 pacientes com infecção confirmada para COVID-19, destes 53\% desenvolveram LRA durante a internação na UTI que foi em média de 13 dias. A prevalência de LRA foi em pacientes do sexo masculino com idade média de 62 anos, com Hipertensão Arterial Sistêmica e Diabetes Mellitus de comorbidades prévias, sendo a taxa de mortalidade de 67,95\%. Observamos a presença de hipercalemia, anemia e aumento dos níveis de creatinina e ureia nesses pacientes. Outra questão analisada foi o uso de antimicrobianos nefrotóxicos, em que a polimixina $(83,01 \%)$ foi a mais utilizada, seguida pela classe dos aminoglicosídeos (73,58\%). Visto a necessidade de ajuste dos antimicrobianos, foi analisado que dos pacientes que utilizaram os aminoglicosídeos, apenas 26,65\% receberam ajuste renal. De acordo com os dados encontrados, pudemos ver que a COVID-19 auxiliou no desenvolvimento de LRA, pois obtivemos resultados de alta taxa de incidência e grande número de óbitos, por outro lado, o grande uso de antibióticos no curso da doença e a falta de ajuste renal dos mesmos também podem auxiliar no desenvolvimento de lesões renais.
\end{abstract}

Palavras-chave: Injúria renal aguda; Antibacterianos; COVID-19.

\begin{abstract}
The objective of this research was to characterize the profile of patients who developed AKI, analyze laboratory tests, classify according to stages and analyze the adjustment of dosage of antimicrobials. This is a cross-sectional, analytical and retrospective study, whose data collection was carried out with information contained in the MVPEP ${ }^{\circ}$ Electronic Medical Record System. During the study period, 100 patients were found with confirmed infection for COVID-19, of which 53\% developed AKI during ICU stay, which was an average of 13 days. The prevalence of AKI was in male patients with a mean age of 62 years, with Systemic Arterial Hypertension and Diabetes Mellitus of previous comorbidities, with a mortality rate of $67.95 \%$. We observed the presence of hyperkalemia, anemia and increased levels of creatinine and urea in these patients. Another issue analyzed was the use of nephrotoxic antimicrobials, in which polymyxin $(83.01 \%)$ was the most used, followed by the aminoglycoside class $(73,58 \%)$. Given the need to adjust antimicrobials, it was analyzed that of the patients who used aminoglycosides, only $26.65 \%$ received renal adjustment. According to the data found, we could see that COVID-19 helped in the development of AKI, as we obtained results of a high incidence rate and a large number of deaths, on the other hand, the large use of antibiotics in the course of the disease and the lack of renal adjustment of these can also help in the development of kidney injury.
\end{abstract}

Keywords: Acute kidney injury; Antibacterials; COVID-19.

\section{Resumen}

El objetivo de esta investigación fue caracterizar el perfil de los pacientes que desarrollaron LRA, analizar las pruebas de laboratorio, clasificar por estadios y analizar el ajuste de dosis de antimicrobianos. Se trata de un estudio transversal, analítico y retrospectivo, cuya recogida de datos se realizó con información contenida en el Sistema de 
Research, Society and Development, v. 11, n. 1, e27411124961, 2022

(CC BY 4.0) | ISSN 2525-3409 | DOI: http://dx.doi.org/10.33448/rsd-v11i1.24961

Historia Clínica Electrónica MVPEP®. Durante el período de estudio, se encontraron 100 pacientes con infección confirmada por COVID-19, de los cuales el 53\% desarrolló LRA durante la estadía en la UCI, que fue un promedio de 13 días. La prevalencia de LRA fue en pacientes varones con una edad media de 62 años, con Hipertensión Arterial Sistémica y Diabetes Mellitus de comorbilidades previas, con una tasa de mortalidad del 67,95\%. Observamos la presencia de hiperpotasemia, anemia y niveles elevados de creatinina y urea en estos pacientes. Otro tema analizado fue el uso de antimicrobianos nefrotóxicos, en los que la polimixina $(83,01 \%)$ fue la más utilizada, seguida de la clase de aminoglucósidos (73,58\%). Dada la necesidad de ajuste de antimicrobianos, se analizó que de los pacientes que utilizaron aminoglucósidos, solo el 26,65\% recibió ajuste renal. De acuerdo con los datos encontrados, pudimos ver que COVID-19 ayudó en el desarrollo de LRA, ya que obtuvimos resultados de una alta tasa de incidencia y un gran número de muertes, por otro lado, el gran uso de antibióticos en el curso de la enfermedad y la falta de ajuste renal de estos también pueden ayudar en el desarrollo de daño renal.

Palabras clave: Lesión renal aguda; Antibacterianos; COVID-19.

\section{Introdução}

O novo coronavírus foi relatado pela primeira vez em Wuhan, na China, onde havia vários casos de uma pneumonia desconhecida, que posteriormente foi classificada como a síndrome respiratória aguda grave 2 (SARS-CoV-2). No período de fevereiro de 2020 foi diagnosticado no Brasil o primeiro paciente com a doença, um homem paulista de 61 anos, que havia retornado de uma viagem na Itália. No mesmo período, a situação foi declarada pela Organização Mundial de Saúde (OMS) como uma Emergência de Saúde Pública de Interesse Internacional (ESPII) e mais tarde, em março do mesmo ano, caracterizado como uma pandemia (Teich et al, 2020).

Diante desse cenário, os países se mobilizaram a fornecer o maior número de leitos de seus serviços de saúde para tratar os pacientes com as complicações respiratórias (Moreira, 2020). Contudo, estudos apontam que de 17\% a 35\% dos pacientes adultos internados necessitam de transferência para a unidade de terapia intensiva (UTI) por apresentarem hipoxemia e insuficiência respiratória (Saueressig et al, 2020).

Além da insuficiência respiratória hipoxêmica, a lesão renal aguda (LRA) também foi relatada como uma disfunção orgânica relacionada ao COVID-19 (Lima \& Nunes, 2017). A explicação de alguns estudos sobre essas complicações é que ao ter o caso grave de COVID-19, o organismo apresenta uma resposta imune intensificada, conhecida como "tempestade de citocinas", repercutindo em perda de tolerância periférica aos órgãos, tornando-se antigênico, acompanhado de extensa inflamação e autodestruição tecidual em múltiplos órgãos (Moitinho et al, 2020).

Outros possíveis mecanismos são a formação de trombos de fibrina, a presença de Enzima Conversora de Angiotensina 2 (ACE2) e Proteases de Serina Transmembrana Celulares (TMPRSS2) nas células renais, além de chances de mecanismos inespecíficos como alterações hemodinâmicas, disfunção cardíaca, ventilação mecânica, hipovolemia, uso de drogas nefrotóxicas e sepse favorecer o desenvolvimento de LRA (Zermani et al, 2021; Matos et al, 2021; Pan et al, 2020).

Assim, a LRA é definida como uma danificação da função renal em um determinado período, podendo ser horas ou dias, evidenciando-se pela diminuição do ritmo de filtração glomerular e/ou do volume urinário, ocasionando distúrbios do equilíbrio hidroeletrolítico e ácido-básico (Lima \& Nunes, 2017). Ela pode ser classificada em três estágios (1, 2 e 3) de acordo a elevação da creatinina e/ou diminuição do débito urinário (Kdigo, 2012).

De acordo com Costa et al (2021), 55,9\% dos pacientes internados em UTI evoluíram com algum grau de LRA, sendo que a maioria $(66,7 \%)$ foi classificada como estágio 3. Ao apresentar essa condição, o paciente pode ter sua internação prolongada em 2,4 vezes, o que pode aumentar a morbimortalidade dos pacientes de cuidados intensivos se comparados com aqueles que não apresentaram nenhuma lesão (Lima \& Nunes, 2017).

Pacientes que apresentam doenças crônicas preexistentes como Hipertensão Arterial Sistêmica (HAS), Diabetes Mellitus (DM) e doenças hepáticas podem apresentar uma predisposição a desenvolver uma lesão renal. Também pode 
acontecer de o paciente ser submetido a grandes cirurgias e exposto a septicemia e principalmente, uso de drogas nefrotóxicas (Lima \& Nunes, 2017).

Um tipo de droga nefrotóxica muito utilizada nas UTIs são os antibióticos, que ao serem utilizados por vários dias, como acontece em tratamentos de infecções graves, podem levar ao desenvolvimento de lesão renal dos pacientes. As principais classes de antibióticos nefrotóxicos utilizados são os aminoglicosídeos e as polimixinas, pois além de apresentarem uma nefrotoxicidade importante, são largamente utilizados na área hospitalar, principalmente em UTIs e apresenta maior risco de lesão renal quando a duração do tratamento é aumentada (Oliveira \& Rodrigues, 2019).

Embora alguns dados a respeito da LRA em pacientes com COVID-19 estejam disponíveis na literatura, ainda se faz necessário uma melhor caracterização do perfil dos pacientes que desenvolvem esta infecção nas diversas regiões do globo. Neste sentido, o objetivo deste estudo é caracterizar o perfil de pacientes do Hospital Regional de Mato Grosso do Sul que desenvolveram a lesão renal e descrever quais foram os fatores que podem ter colaborado para o surgimento desta lesão.

\section{Metodologia}

Trata-se de um estudo transversal, analítico e retrospectivo (Pereira, Shitsuka, Parreira, \& Shitsuka, 2018), em que foi analisado os exames laboratoriais e as prescrições médicas, com o intuito de identificar a presença de lesão renal aguda nos pacientes internados na Unidade de Terapia Intensiva (UTI) do Hospital Regional de Mato Grosso do Sul (HRMS) e que fizeram uso de antimicrobianos.

A pesquisa foi desenvolvida no período de julho a dezembro de 2020 na UTI adulta 1, composta por um total de 10 leitos onde são atendidos pacientes críticos com COVID-19. As prescrições médicas e exames laboratoriais que foram analisados ficam disponível eletronicamente no Sistema de Prontuário Eletrônico MVPEP® para consulta.

A amostra foi composta pelas prescrições e prontuários médicos de pacientes internados na UTI adulta no período de julho a dezembro de 2020.

Foram incluídos neste estudo pacientes com idade $\geq 18$ anos, resultado de RT-PCR e teste rápido para COVID-19 positivo, tempo de permanência na UTI $\geq$ que 48 horas, sem lesão renal pré-existente e com prescrição de pelo menos um antimicrobiano.

Foram excluídos da pesquisa pacientes que já apresentavam lesões renais prévias, que estivessem em cuidados paliativos, que evoluíram para alta ou óbito em menos de 48 horas da admissão na UTI, com resultado negativo em RT-PCR e que não tinham prescrição de antimicrobianos. O estudo foi realizado após a aprovação do Comitê de Ética em Pesquisa da Universidade Anhanguera-UNIDERP (CEP/UNIDERP) através do parecer nº 4.847 .130 em 14 de Julho de 2021 (CAAE 47766821.0.0000.5161).

Em termos de análise de dados, a normalidade dos dados foi analisada pelo teste de Kolmogorov-Smirnov. As variáveis categóricas foram expressas em frequência absoluta (n) e frequência relativa (\%). As variáveis contínuas foram expressas em mediana e distribuição mínima e máxima para dados não normais e média e desvio padrão para dados normais. A comparação entre as variáveis não normais foi realizada pelo teste não paramétrico Mann-Whitney (duas amostras de dados) e Kruskal-Wallis com pós-teste de Dunn (três ou mais amostras de dados. A comparação de dados normais foi realizada pelo tStudent não pareado. Quanto a correlação dos dados, utilizou-se o teste de Spearman. As análises foram realizadas nos softwares GraphPad Instat 3 e Graph Pad Prism 9, sendo considerado significante p <0,05. 


\section{Resultados}

No período de julho a dezembro de 2020, foram admitidos em um setor de UTI adulta, 182 pacientes críticos, sendo 106 pacientes com COVID-19. Destes, 6 pacientes foram excluídos do estudo devido a alta ou óbito em um período menor que 48 horas da admissão na UTI e presença de Doença Renal Crônica (DRC). Dessa forma, participaram do estudo 100 pacientes graves infectados com Sars-CoV-2 internados na UTI.

Na Tabela 1, está apresentada a distribuição dos pacientes quanto a presença ou ausência de LRA de acordo com a classificação pelos critérios da KDIGO e as características clínicas e demográficas. Na população de estudo, a incidência de LRA foi de 53\%, predominando o sexo masculino (52,83\%). Na estratificação dos estágios de injúria renal, $64 \%$ evoluíram para o estágio 3 da doença. O sexo masculino também foi o mais frequente nesse estágio, comprometendo $55,88 \%$ dos pacientes.

Tabela 1: Distribuição das variáveis categóricas da população de estudo estratificados pela injúria renal aguda. Valores expressos em n $(\%)$.

\begin{tabular}{|c|c|c|c|c|c|}
\hline \multirow[b]{2}{*}{ Variáveis } & \multirow{2}{*}{$\begin{array}{c}\text { Sem LRA } \\
(n=47)\end{array}$} & \multirow{2}{*}{$\begin{array}{c}\text { Com LRA } \\
(n=53)\end{array}$} & \multicolumn{3}{|c|}{ Estágio da LRA } \\
\hline & & & $\begin{array}{c}\text { Estágio } 1 \\
\qquad(\mathbf{n}=\mathbf{3})\end{array}$ & $\begin{array}{c}\text { Estágio } 2 \\
(\mathrm{n}=16)\end{array}$ & $\begin{array}{c}\text { Estágio } 3 \\
(\mathbf{n}=\mathbf{3 4})\end{array}$ \\
\hline \multicolumn{6}{|l|}{ Sexo } \\
\hline Feminino & $24(51,06)$ & $25(47,16)$ & $2(66,6)$ & $8(50)$ & $15(44,11)$ \\
\hline Masculino & $23(48,93)$ & $28(52,83)$ & $1(33,3)$ & $8(50)$ & $19(55,88)$ \\
\hline \multicolumn{6}{|l|}{ Comorbidades } \\
\hline $\begin{array}{l}\text { Hipertensão } \\
\text { arterial }\end{array}$ & $21(44,68)$ & $34(64,15)$ & $2(66,6)$ & $10(62,5)$ & $22(64,7)$ \\
\hline Diabetes & $12(25,53)$ & $20(37,73)$ & $2(66,6)$ & $6(37,5)$ & $12(35,29)$ \\
\hline Obesidade & $7(14,89)$ & $11(20,75)$ & $1(33,3)$ & $2(12,5)$ & $8(23,52)$ \\
\hline DPOC* & $1(2,12)$ & $4(7,54)$ & - & - & $4(11,76)$ \\
\hline Asma & $1(2,12)$ & $3(5,66)$ & - & $1(6,25)$ & $2(5,88)$ \\
\hline Hipotireoidismo & $1(2,12)$ & 2 & $1(33,3)$ & $1(6,25)$ & - \\
\hline Outros** & $2(4,25)$ & 6 & $1(33,3)$ & - & $5(14,7)$ \\
\hline \multicolumn{6}{|l|}{ Desfecho } \\
\hline Alta & $25(53,19)$ & & $1(33,3)$ & $5(31,25)$ & $5(14,7)$ \\
\hline Transferência & $9(19,14)$ & & - & $1(6,25)$ & $4(11,76)$ \\
\hline Óbito & $13(27,65)$ & & $2(66,6)$ & $10(62,5)$ & $25(73,52)$ \\
\hline
\end{tabular}

*DPOC: Doença pulmonar obstrutiva crônica. **Sem LRA: tabagismo; urolitíase.

**Com LRA: Cardiopatia; esquizofrenia; poliomielite infantil; arritmia; dislipidemia câncer de mama.

**Estágio 1: câncer de mama; **Estágio 3: Cardiopatia; esquizofrenia; poliomielite infantil; arritmia; dislipidemia.

Fonte: Autores. 


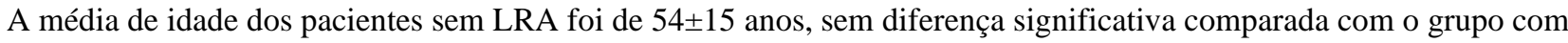
LRA (62 \pm 11 anos). Embora não tenha apresentado diferença significativa da idade entre os estágios de LRA, observa-se que os pacientes em estágio 3 apresentam idade mais avançada em comparação aos demais estágios, sobretudo, em relação ao estágio 1.

A hipertensão arterial e a diabetes foram as comorbidades mais encontradas no grupo com LRA, representando $64 \%$ e $37 \%$ dos pacientes, respectivamente. As mesmas comorbidades foram as mais prevalentes no estágio 3 da doença.

Na população geral, 49\% (49) dos pacientes evoluíram para óbito. No grupo dos pacientes sem LRA houve 27,6\% (13) óbitos ao passo que no grupo com LRA 69,8\% (37) morreram devido às complicações. De 36\% das altas na população geral, $69 \%$ foram direcionadas para os pacientes que não desenvolveram LRA. A maior taxa de mortalidade no estudo foi observada nos pacientes com LRA em estágio 3 (73,52\%).

As Figuras 1 e 2 mostram a diferença significativa dos valores de creatinina e ureia na admissão da UTI entre os pacientes com e sem LRA. Consequentemente, os pacientes com alterações renais apresentaram valores das escórias nitrogenadas mais elevadas. Os pacientes sem LRA apresentaram valores de creatinina na admissão de $0,92 \mathrm{mg} / \mathrm{dL}(0,4-2,7)$ e ureia de $37 \mathrm{mg} / \mathrm{dL}(10$ - 146). Enquanto os pacientes com LRA apresentaram valores de creatinina de $1,03 \mathrm{mg} / \mathrm{dL}(0,74-2,91)$ e ureia de $43 \mathrm{mg} / \mathrm{dL}(16-198)$.

Figura 1: Comparação dos valores de creatinina entre pacientes sem e com lesão renal aguda na admissão a UTI. Valores expressos em mediana e distribuição mínima e máxima (Teste de Mann-Whitney).

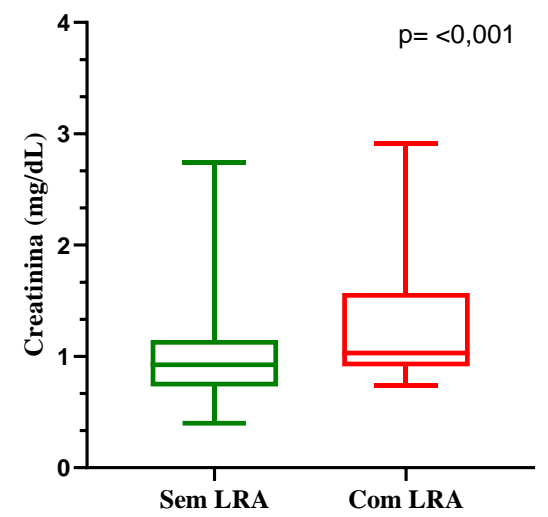

Fonte: Autores. 
Figura 2: Comparação dos valores de ureia entre pacientes sem e com lesão renal aguda na admissão a UTI. Valores expressos em mediana e distribuição mínima e máxima (Teste de Mann-Whitney).

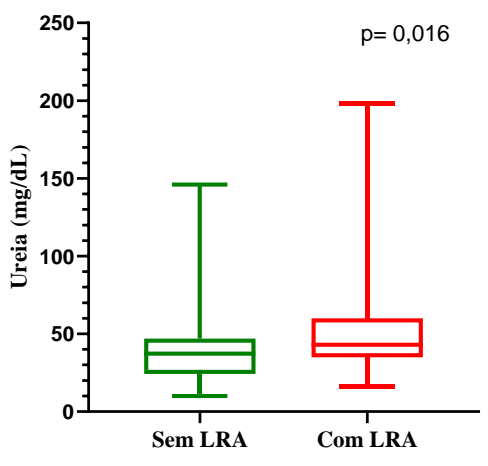

Fonte: Autores.

Ao longo da internação, os pacientes com LRA apresentaram valores de creatinina e ureia significativamente mais elevados em relação ao período da admissão ( $\mathrm{p}<0,001$ ), sugerindo piora do quadro renal. A mediana da creatinina aumentou para $1,79 \mathrm{mg} / \mathrm{dL}(0,64-6,84)$ e a ureia de $96 \mathrm{mg} / \mathrm{dL}(42$ - 177). Já nos pacientes sem LRA, as escórias nitrogenadas apresentaram um declínio importante em relação a admissão $(\mathrm{p}=0,001)$. A mediana da creatinina e ureia foi de $0,75 \mathrm{mg} / \mathrm{dL}$ $(0,54-1,23)$ e $45 \mathrm{mg} / \mathrm{dL}(26-91)$, respectivamente.

A idade parece ser um dos fatores associados a elevação de ureia nos pacientes com LRA do nosso estudo. Na correlação de Spearman, observamos uma correlação positiva entre a idade e os valores de ureia, no qual ambos se elevam concomitantemente $(r=0,393 ; p=0,023)$. Essa característica não foi observada na creatinina $(r=0,212 ; p=0,134)$.

O tempo de internação na UTI foi mais elevado nos pacientes com LRA que necessitaram de assistência em média de 13 (1 - 54) dias. Em contrapartida, o grupo sem LRA permaneceu em cuidados intensivos em cerca de 10 (1 - 26) dias, sendo significativamente menor. Além disso, não houve correlação da idade com o período de permanência na UTI ( $\mathrm{r}=-0,160$; $\mathrm{p}=0$, 458).

$\mathrm{Na}$ Tabela 2, variáveis laboratoriais foram comparadas entre pacientes com e sem lesão renal durante o período de cuidados intensivos. Durante esse tempo, os pacientes com LRA apresentaram uma hipercalemia importante em relação ao outro grupo, sobretudo, nos pacientes com LRA em estágio 3. Além disso, a anemia foi observada em ambos, porém, os pacientes com LRA apresentaram anemia mais significativa. Não houve diferença significativa no quadro de anemia entre os estágios de LRA. 
Tabela 2: Parâmetros laboratoriais avaliados nos pacientes internados na UTI com COVID-19 (n=100). Valores expressos em mediana e distribuição mínima e máxima. (Teste de Mann-Whitney e Kruskal-Wallis com pós-teste de Dunn)

\begin{tabular}{|c|c|c|c|c|c|c|c|}
\hline \multirow[b]{2}{*}{$\begin{array}{l}\text { Parâmetros } \\
\text { laboratoriais }\end{array}$} & \multirow[b]{2}{*}{$\begin{array}{c}\text { Sem LRA } \\
(n=47)\end{array}$} & \multirow{2}{*}{$\begin{array}{c}\text { Com LRA } \\
\qquad(n=53)\end{array}$} & \multirow[b]{2}{*}{ Valor de $p$} & \multicolumn{4}{|c|}{ Estágio da LRA } \\
\hline & & & & $\begin{array}{c}\text { Estágio } 1 \\
(\mathbf{n}=3)\end{array}$ & $\begin{array}{c}\text { Estágio } 2 \\
(n=16)\end{array}$ & $\begin{array}{c}\text { Estágio } 3 \\
(n=34)\end{array}$ & $\begin{array}{c}\text { Valor de } \\
\text { p }\end{array}$ \\
\hline $\begin{array}{l}\text { Hemoglobina } \\
(\mathrm{g} / \mathrm{dL})\end{array}$ & $\begin{array}{c}11,08(8,47- \\
15,22)\end{array}$ & $\begin{array}{c}10,1(5,54- \\
13,9)\end{array}$ & 0,014 & $\begin{array}{c}10,5(9,58- \\
13,09)\end{array}$ & $\begin{array}{c}11,04(8,54- \\
14,31)\end{array}$ & $\begin{array}{c}9,85(8,29- \\
13,91)\end{array}$ & 0,197 \\
\hline $\begin{array}{l}\text { Potássio } \\
\text { (mmol/L) }\end{array}$ & $\begin{array}{c}4,12(3,07- \\
4,70)\end{array}$ & $\begin{array}{c}4,36(3,18- \\
5,50)\end{array}$ & 0,009 & $\begin{array}{c}3,98(3,52- \\
4,11)\end{array}$ & $\begin{array}{c}4,31(3,4- \\
5,5)\end{array}$ & $\begin{array}{c}4,57(3,66- \\
5,31)\end{array}$ & 0,072 \\
\hline $\begin{array}{l}\text { Sódio } \\
\text { (mmol/L) }\end{array}$ & $\begin{array}{c}143(135- \\
152)\end{array}$ & $\begin{array}{c}142,5(133- \\
153)\end{array}$ & 0,425 & $\begin{array}{c}149(148- \\
153)\end{array}$ & $\begin{array}{c}143(136- \\
154)\end{array}$ & $\begin{array}{c}143(133- \\
151)\end{array}$ & 0,045 \\
\hline $\begin{array}{l}\text { Creatinina } \\
(\mathrm{mg} / \mathrm{dL})\end{array}$ & $\begin{array}{c}0,75(0,54- \\
1,23)\end{array}$ & $\begin{array}{c}1,79(0,64- \\
6,84)\end{array}$ & $<0,001$ & $\begin{array}{c}1,02(0,88- \\
1,10)\end{array}$ & $\begin{array}{c}1,34(0,78- \\
2,29)\end{array}$ & $\begin{array}{c}2,09(0,86- \\
6,84)\end{array}$ & $<0,001$ \\
\hline $\begin{array}{l}\text { Ureia } \\
(\mathrm{mg} / \mathrm{dL})\end{array}$ & $45(26-91)$ & $96(42-177)$ & $<0,001$ & $79(62-85)$ & $\begin{array}{c}79,2(42- \\
150)\end{array}$ & $\begin{array}{c}107,3(47,4- \\
177)\end{array}$ & 0,0018 \\
\hline
\end{tabular}

Fonte: Autores.

Os agentes nefrotóxicos utilizados nos pacientes com LRA na UTI estão descritos na tabela 3 . O antibiótico nefrotóxico mais utilizado foi a polimixina $(83,01 \%)$, seguido da amicacina $(52,83 \%)$. Em menores frequências, aparecem principalmente o sulfametoxazol + trimetoprima $(5,66 \%)$ e vancomicina $(3,77 \%)$.

Visto que a amicacina e a gentamicina são da classe dos aminoglicosídeos, associamos esses dois antimicrobianos para a análise comparativa com as variáveis do estudo (eletrólitos, ureia, creatinina e hemoglobina). Foi observado que aproximadamente $64 \%$ dos pacientes que utilizaram essa classe necessitavam de ajuste na posologia, no entanto, menos da metade foram ajustados (Tabela 3). 
Tabela 3: Antibióticos nefrotóxicos utilizados em pacientes com COVID-19 que evoluíram com lesão renal aguda.

\begin{tabular}{|c|c|c|c|c|c|c|}
\hline & \multirow{2}{*}{$\begin{array}{l}\text { Frequência } \\
\text { absoluta (n) }\end{array}$} & \multirow{2}{*}{$\begin{array}{l}\text { Frequência } \\
\text { relativa (\%) }\end{array}$} & \multicolumn{2}{|c|}{ Ajuste de antibiótico (\%) } & \multicolumn{2}{|c|}{ Necessário ajuste (\%) } \\
\hline & & & Sim & Não & Sim & Não \\
\hline \multicolumn{7}{|l|}{ Antibióticos } \\
\hline Polimixina & 44 & 83,01 & - & 100 & 11,62 & 88,37 \\
\hline Amicacina & 28 & 52,83 & 35,2 & 64,28 & 64,9 & 35,71 \\
\hline Azitromicina & 20 & 37,73 & - & - & - & - \\
\hline Claritromicina & 16 & 30,18 & - & - & - & - \\
\hline Gentamicina & 11 & 20,75 & 18,1 & 81,8 & 63,6 & 36,3 \\
\hline $\begin{array}{l}\text { Sulfametoxazol } \\
\text { +Trimetoprima }\end{array}$ & 3 & 5,66 & - & - & - & - \\
\hline Vancomicina & 2 & 3,77 & 50 & 50 & 50 & 50 \\
\hline
\end{tabular}

Fonte: Autores.

Os efeitos dos aminoglicosídeos nefrotóxicos nos marcadores renais de pacientes com LRA. Do total de 53 pacientes com LRA, $28(52,83 \%)$ utilizaram a amicacina e apenas $11(20,75 \%)$ trataram com gentamicina. Quanto a ureia e creatinina, não houve diferença significativa na comparação de pacientes que fizeram ou não uso dos aminoglicosídeos durante o tratamento.

Quanto aos eletrólitos, também não foi observado diferença entre o sódio e os grupos que utilizaram ou não os aminoglicosídeos. Porém, observa-se que os pacientes que utilizaram gentamicina apresentaram aumento nos valores de potássio. Além disso, os pacientes que fizeram uso dos aminoglicosídeos apresentaram uma anemia mais relevante em comparação aos que não fizeram uso dessa classe.

\section{Discussão}

Analisando os primeiros países que foram infectados pela COVID-19, temos a China em um estudo realizado no início da pandemia, em que a LRA já estava presente tanto na admissão quanto durante a internação hospitalar. Embora os resultados mostraram que a incidência de LRA nos pacientes foi de 5,1\%, menor que em nosso estudo (53\%), os autores relatam que o risco de morte intra-hospitalar é significantemente maior. Dos pacientes que apresentaram lesão renal, a maioria se enquadrava no estágio 3 (2\%) como em nossa pesquisa, com 64\% dos pacientes classificados no mesmo estágio (CHENG et al, 2020).

Um estudo da França e Espanha, em março de 2020, avaliou 9 UTI’s, em que foram incluídos 211 pacientes, 105 (58\%) desenvolveram LRA e 55 (26\%) foram classificados como estágio 3 em até 7 dias após a admissão na UTI. Dentre os pacientes do estágio 3, as comorbidades mais frequentes foram HAS com 48\% e DM com 35\%, sendo que o sexo masculino predominou (82\%), com idade média de 63 anos e a mortalidade foi de $24 \%$ no $28^{\circ}$ dia de internação (CHAIBI et al, 2020). Sendo que em nosso estudo encontramos um número de incidência parecido, sendo ele 53\%, com $64 \%$ classificados no estágio 3, valor maior se comparado ao estudo da França e Espanha. Maioria dos pacientes do sexo masculino (51\%), menor que na pesquisa encontrada na literatura, com média de 62 anos, parecida com a do estudo, com predomínio de HAS (64\%) e DM 
Research, Society and Development, v. 11, n. 1, e27411124961, 2022

(CC BY 4.0) | ISSN 2525-3409 | DOI: http://dx.doi.org/10.33448/rsd-v11i1.24961

(37\%) como comorbidades, sendo que HAS foi vista com mais frequência em nosso estudo e taxa de mortalidade de 67,9\% quase três vezes maior que no estudo de Chaibi et al (2020).

Um estudo realizado por Costa et al (2020), baseado nos pacientes que apresentavam COVID-19 no Brasil, demonstrou uma incidência de LRA de 55,9\%, sendo que destes 66,7\% foram classificados como estágio 3. Nas características gerais dos pacientes com LRA, a maioria era do sexo masculino $(68,4 \%)$, com idade média de 67,4 anos. Como comorbidade houve predomínio de HAS presente em $66,7 \%$ dos pacientes, seguido pela DM com 26,3\%. De acordo com o tempo de permanência, este foi maior nos pacientes que apresentaram lesão renal, com média de 15 dias e nos pacientes do estágio 3 de 18 dias. Comparado ao nosso estudo, muitas características foram parecidas, diferindo na porcentagem de DM encontrada nos pacientes, que em nosso estudo foi $37 \%$, maior que a vista na literatura e a permanência de pacientes com LRA na UTI foi de 13 dias em média em nossa pesquisa. A idade acima de 65 anos, sexo masculino e manifestação grave da COVID-19 estavam associados a maior mortalidade hospitalar, mesmo a mortalidade geral do estudo sendo 16,1\%, baixa se comparada ao nosso estudo que foi de $67,9 \%$.

Outra pesquisa brasileira entre março e maio de 2020, mostra que de 201 pacientes, 101 (50,2\%) desenvolveram LRA, sendo que 67 (66,3\%) eram do sexo masculino com idade média de 73 anos. As comorbidades mais presentes foram HAS $(58,4 \%)$ e DM $(38,6 \%)$ e os antibióticos foram utilizados em $99 \%$ dos pacientes. A média de internação foi de 13,5 dias na UTI e a mortalidade foi de 41,6\%. Outras variáveis utilizadas para avaliar LRA, foram a creatinina, hemoglobina e os eletrólitos (sódio e potássio), sendo que o resultado delas foram de 1,27mg/dL, 12,5g/dL, 138mEq/L e 4,2mEq/L respectivamente na admissão (Doher et al, 2020).

Comparando com nosso estudo, a média de idade encontrada foi maior que a nossa (62 anos), a nossa taxa de mortalidade foi maior com 67,9\%. A respeito dos eletrólitos, encontramos valores de 1,03mg/dL de creatinina na admissão e durante a internação mediana de 1,79mg/dL de creatinina, 10,1 g/dL de hemoglobina, 142,50mmol/L de sódio e 4,36mmol/L de potássio, sendo que o valor de creatinina aumentou durante a internação, a anemia em nossos pacientes foi mais significativa, o potássio e sódio não tiveram diferenças relevantes se comparado a pesquisa de Doher et al (2020).

Diversas pesquisas apresentam valores de creatinina para avaliar a lesão renal nos pacientes, pois a creatinina é o biomarcador padrão para diagnóstico de LRA (Peres et al, 2013). Também foi utilizado no presente estudo o valor de ureia, porém poucos trabalhos utilizam esse biomarcador, pois ele pode ser elevado por outras causas, não só por lesão renal. Alguns fatores não associados a LRA são hemorragias gastrointestinais, dieta rica em proteínas e terapia com corticoides, este último sendo muito utilizado no tratamento da COVID-19 e em choques dentro da UTI (Abensur, 2011).

A ureia se eleva independentemente dos níveis séricos de creatinina por consequência de processos patológicos distintos, como por exemplo na insuficiência cardíaca (Abensur, 2011). Em nosso estudo utilizamos a ureia, pois é um biomarcador solicitado diariamente nos exames laboratoriais dos pacientes da UTI, que em associação com a creatinina nos permite avaliar a função renal.

Carelli et al (2020), em uma pesquisa de alterações laboratoriais em pacientes com COVID-19, observou alterações de ureia e creatinina na admissão dos pacientes da UTI, com média de $86,9 \mathrm{mg} / \mathrm{dL}$ e 2,03mg/dL respectivamente. Durante a internação, esses valores continuaram a aumentar, alcançando médias de 250mg/dL e 3,93mg/dL. Podemos comparar essas variáveis com as do nosso estudo, em que observamos valores mais baixos com mediana da ureia de $37 \mathrm{mg} / \mathrm{dL}$ na admissão e $96 \mathrm{mg} / \mathrm{dL}$ durante a internação e a creatinina de $0,92 \mathrm{mg} / \mathrm{dL}$ na admissão e $1,79 \mathrm{mg} / \mathrm{dL}$ durante a internação.

Outros fatores importantes encontrados em Carelli et al (2020) foi o aumento dos valores de potássio com média de $5,44 \mathrm{mmol} / \mathrm{L}$ e diminuição da hemoglobina com média de $8,059 \mathrm{~g} / \mathrm{dL}$ comparando ao nosso estudo, onde foi encontrado valores 
Research, Society and Development, v. 11, n. 1, e27411124961, 2022

(CC BY 4.0) | ISSN 2525-3409 | DOI: http://dx.doi.org/10.33448/rsd-v11i1.24961

de potássio com máxima de 5,50mmol/L e mínima de hemoglobina de 5,54g/dL, este último valor bem abaixo do ideal. Já a idade encontrada foi maior que 65 anos, diferindo pouco ao comparar com nossa pesquisa que a idade foi de 62 anos

A hipercalemia pode ser explicada, pois o íon de potássio é eliminado em $90 \%$ nos rins e a diminuição da filtração glomerular ou a inativação renal leva a um aumento desse íon na corrente sanguínea. Sendo assim, é um importante marcador da função renal que deve ser avaliado periodicamente (Saloio et al, 2019). Já a hemoglobina quando sofre diminuição, faz com que o paciente necessite de transfusão sanguínea, o que gera uma resposta pró-inflamatória, prejudicando a distribuição de oxigênio ao parênquima renal aumentando o estresse oxidativo nos tecidos. Isto em associação com comorbidades préexistentes aumenta a predisposição do paciente desenvolver LRA (Duarte et al, 2020).

Outra explicação da anemia em pacientes com LRA é a diminuição da produção de eritropoetina. Mesmo podendo ser gerada por muito tecidos, a eritropoetina necessária para a eritropoiese é produzida pelas células epiteliais próximas aos túbulos renais. Contudo, quando a Taxa de Filtração Glomerular (TFG) é diminuída e há alterações na função renal, esse hormônio também reduz (Ribeiro et al, 2021).

Um estudo feito por Oliveira e Rodrigues (2019), realizado na mesma UTI e hospital da presente pesquisa, no período de abril a setembro de 2017, apresentou um perfil demográfico de pacientes com maior incidência de LRA no sexo masculino $(67,56 \%)$ com média de idade de 56 anos, com período de internação maior que 10 dias. Mesmo os pacientes não tendo como doença de base o COVID-19, a incidência de LRA foi de 67,27\%, valor maior se comparado ao dessa pesquisa, porém com mortalidade inferior $(35,13 \%)$. Observamos que o perfil do hospital permanece o mesmo se comparado com um período anterior a pandemia, porém a mortalidade dos pacientes com COVID-19 e LRA observados nesse estudo foi maior, podendo mostrar que a forma grave do COVID-19 pode ter complicações severas, com taxa de mortalidade alta.

A LRA também pode se desenvolver quando são utilizados alguns medicamentos, principalmente os antibióticos. Nesse estudo vimos quais deles foram utilizados e da classe dos aminoglicosídeos quais foram feitos os devidos ajustes.

Sendo assim, os antimicrobianos mais utilizados nos pacientes críticos da pesquisa foram as polimixinas $(83,01 \%)$, seguido dos aminoglicosídeos (73,58\%). A primeira classe mais utilizada possui efeitos neuro e nefrotóxicos, são excretados pelos rins com até $60 \%$ do fármaco presente na urina. Estudos em pacientes críticos são escassos, no entanto, mais que $50 \%$ dos pacientes que a utilizam evoluem com LRA (Coelho, 2018).

A classe dos aminoglicosídeos abrange os antibióticos amicacina e gentamicina. A amicacina por sua vez, apresenta efeitos de nefrotoxicidade, neurotoxicidade e ototoxicidade, enquanto que a gentamicina apresenta apenas nefrotoxicidade, porém é a mais nefrotóxica da classe. Estes efeitos podem ser diminuídos quando as doses desses medicamentos sejam realizadas em um único período no dia (Cunha et al, 2017).

Em nosso estudo observamos que quem utilizou os aminoglicosídeos apresentou anemia e quem fez uso apenas a gentamicina manifestou aumento nos valores de creatinina e potássio se comparado aos pacientes que não utilizaram essa classe, mesmo os dados não terem se mostrados significantes nos testes estatísticos. Não foram encontrados na literatura estudos que relacionaram os antibióticos com os valores séricos de hemoglobina e os eletrólitos (potássio e sódio) como nesse estudo para comparação.

Com a LRA presente, muitos antimicrobianos sofrem alteração na concentração plasmática, sendo necessário exames de rotina tanto para avaliação da função renal quanto para auxiliar em um possível ajuste desses medicamentos, contribuindo assim para a redução da nefrotoxicidade (Marques et al, 2020).

Para nos guiar no ajuste dos antibióticos, utilizamos o protocolo do hospital, que é o guia prático dos médicos (anexo 1). Nele podemos ver qual a posologia recomendada de acordo com o valor da TFG, o qual nos mostra quanto de sangue está sendo filtrado nos rins, ou se necessita de dose adicional após hemodiálise quando o paciente se classifica no estágio 3 . Sendo 
Research, Society and Development, v. 11, n. 1, e27411124961, 2022

(CC BY 4.0) | ISSN 2525-3409 | DOI: http://dx.doi.org/10.33448/rsd-v11i1.24961

assim, quando analisamos o ajuste dos aminoglicosídeos, observamos que $64,25 \%$ dos pacientes que utilizaram essa classe de antimicrobianos necessitavam de ajuste na posologia, porém somente $26,65 \%$ das prescrições foram ajustadas. Não foram encontrados na literatura estudos que analisaram se os antibióticos foram ajustados conforme função renal para comparação.

A dificuldade encontrada nessa pesquisa foi saber a real causa das lesões renais encontradas, se somente a COVID-19 causou todas elas ou se os antibióticos auxiliaram nesse acontecimento. Porém de acordo com os dados encontrados podemos ver que a COVID-19 auxiliou no desenvolvimento de LRA, pois os resultados apresentaram alta taxa de incidência e elevado número de óbitos, por outro lado, o uso de antibióticos relacionado a infecções hospitalares e a falta de ajuste renal dos mesmos também pode ter auxiliado e/ou complicado a situação dos pacientes.

\section{Conclusão}

Com base na população do estudo, observamos que a incidência de LRA foi alta em vários estudos, principalmente no nosso, sendo o perfil desses pacientes predominantemente do sexo masculino e de maior faixa etária, que apresentavam principalmente HAS e DM como comorbidades.

O perfil demográfico e clínico dos pacientes reflete a gravidade da população estudada e demonstra que o COVID-19 favoreceu a alta taxa de incidência dos casos pela inflamação causada nos tecidos e órgãos.

O uso de antimicrobianos em pacientes com COVID-19 auxiliam na evolução das complicações renais, pois além deles serem nefrotóxicos, a não realização do ajuste renal faz com que essa lesão piore e cause mais danos ao paciente, podendo este evoluir para um desfecho desfavorável, conforme alta taxa de mortalidade encontrada em nossa pesquisa.

Podemos observar também a importância da monitorização dos eletrólitos como o potássio e dos valores de hemoglobina além do biomarcador da creatinina no controle da LRA, pois eles também auxiliam no acompanhamento da evolução ou melhora do quadro dos pacientes. São variáveis que também se alteram conforme o estágio da lesão e nos mostram a necessidade da TRS.

O estudo demonstrou a porcentagem de ajuste renal realizado nos antibióticos utilizados, evidenciando que a presença do farmacêutico é indispensável para auxiliar nos tratamentos. Para melhorar o sistema renal dos pacientes críticos e demonstrar essa melhora em números, há necessidade de analisar as taxas de adesão ao ajuste de posologia quando o pesquisador consegue realizar intervenções durante a pesquisa e mostrar a diferença na adesão do ajuste.

\section{Referências}

Abensur, H. (2011). book: Biomarcadores na Nefrologia. Roche Diagnóstica Brasil Ltda. São Paulo.

Carelli, G. Z., de Brito Carvalho, B. R., Dal Molin, B., Duarte, C. R., Martins, G. K. F., Wendt, G. W., ... \& Ferreto, L. E. D. (2020). Alterações laboratoriais em pacientes com COVID-19. Research, Society and Development, 9(12), e30191211115-e30191211115.

Chaibi, K., Dao, M., Pham, T., Gumucio-Sanguino, V. D., Di Paolo, F. A., Pavot, A., ... \& Gaudry, S. (2020). Severe acute kidney injury in patients with COVID-19 and acute respiratory distress syndrome. American Journal of Respiratory and Critical Care Medicine, 202(9), 1299-1301.

Cheng, Y., Luo, R., Wang, K., Zhang, M., Wang, Z., Dong, L., ... \& Xu, G. (2020). Kidney disease is associated with in-hospital death of patients with COVID-19. Kidney international, 97(5), 829-838.

Costa, R. L. D., Sória, T. C., Salles, E. F., Gerecht, A. V., Corvisier, M. F., Menezes, M. A. D. M., ... \& Simvoulidis, L. F. N. (2021). Lesão renal aguda em pacientes com Covid-19 de uma UTI no Brasil: incidência, preditores e mortalidade hospitalar. Brazilian Journal of Nephrology.

Cunha, G. R., da Rocha, R. A. R., Andreu, R. E., \& Ferreira, L. B. manejo da nefrotoxicidade por aminoglicosídeos no setor de emergência: relato de caso.

Matos, A. C. G., de Andrade, T. V., Noronha, N. C. M., Lima, T. B., do Nascimento, C. I. S., Santos, A. C. M., ... \& de Jesus, C. V. F. (2021). Lesão renal aguda na COVID-19 e abordagem multiprofissional. Research, Society and Development, 10(3), e53310313436-e53310313436.

Doher, M. P., De Carvalho, F. R. T., Scherer, P. F., Matsui, T. N., Ammirati, A. L., Da Silva, B. C., ... \& de Souza Durão, M. (2021). Acute kidney injury and renal replacement therapy in critically ill COVID-19 patients: risk factors and outcomes: a single-center experience in Brazil. Blood purification, 50(4-5), 520530 . 
Research, Society and Development, v. 11, n. 1, e27411124961, 2022 (CC BY 4.0) | ISSN 2525-3409 | DOI: http://dx.doi.org/10.33448/rsd-v11i1.24961

Duarte, T. T. P., Lima, W. L., Ribeiro, A. H. S., \& Magro, M. C. S. (2020). Redução de hemoglobina: risco para lesão renal aguda após revascularização do miocárdio. Revista Enfermagem UERJ, 28, 51034.

Khwaja, A. (2012). KDIGO clinical practice guidelines for acute kidney injury. Nephron Clinical Practice, 120(4), c179-c184.

Lima, R. Q. (2018). Lesão renal aguda pós uso de antibióticos. Revista da Faculdade de Medicina de Teresópolis, 2(1), 172-185.

Marques, F. E., de Souza, O. R. P., Bezerra, J. L., de Sousa, J. C., de Andrade, S. M., Cunha, M. A., ... \& de Oliveira, E. H. (2020). Uso de antibióticos na insuficiência renal: necessidade de ajustes posológicos e doses individualizadas. Research, Society and Development, 9(11), e94791110567-e94791110567.

Moitinho, M. S., Belasco, A. G. D. S., Barbosa, D. A., \& Fonseca, C. D. D. (2020). Acute Kidney Injury by SARS-CoV-2 virus in patients with COVID-19: an integrative review. Revista brasileira de enfermagem, 73.

Moreira, R. D. S. (2020). COVID-19: intensive care units, mechanical ventilators, and latent mortality profiles associated with case-fatality in Brazil. Cadernos de saude publica, 36 .

de Oliveira, B. L. C., \& Rodrigues, P. J. N. (2019). Desenvolvimento de lesão renal durante o uso de antibiótico em uma Unidade de Terapia Intensiva. Revista de Saúde Pública de Mato Grosso do Sul, 2(1-2), 32-40.

Pan, X. W., Xu, D., Zhang, H., Zhou, W., Wang, L. H., \& Cui, X. G. (2020). Identification of a potential mechanism of acute kidney injury during the COVID-19 outbreak: a study based on single-cell transcriptome analysis. Intensive care medicine, 46(6), 1114-1116.

Pereira, A. S., Shitsuka, D. M., Parreira, F. J., \& Shitsuka, R. (2018). Metodologia da pesquisa científica.

Ribeiro, J. C., Rodrigues, C. M., \& Neto, A. L. S. (2021). uso de eritropoetina recombinante humana exógena no tratamento da anemia em renais crônicos. Revista Ciência e Saúde On-line, 6(2).

Saloio, M. K., Larissa de Fátima, D. A., Rauber, R., \& Uscocovich, V. (2019). análise dos parâmetros físicos e bioquímicos de pacientes com insuficiência renal crônica em início de terapia renal hemodialítica. fag journal of health (fjh), 1(4), 49-66.

Saueressig, M. G., Hackmann, C. L., da Silva, C. E. S., \& Ferreira, J. (2020). Estimativa de pacientes hospitalizados por COVID-19 em unidade de terapia intensiva no pico da pandemia em Porto Alegre: Estudo com modelo epidemiológico SEIHDR.

Teich, V. D., Klajner, S., Almeida, F. A. S. D., Dantas, A. C. B., Laselva, C. R., Torritesi, M. G., ... \& Cendoroglo, M. (2020). Características epidemiológicas e clínicas dos pacientes com COVID-19 no Brasil. Einstein (São Paulo), 18.

Zermiani, K. (2021). Lesão renal aguda na infecção por SARS-CoV-2: revisão narrativa da literatura (Master's thesis, Florianópolis, SC). 